\title{
La Participación Familiar es un Requisito Imprescindible para una Escuela Inclusiva
}

\section{Family Participation is an Essential Requirement for an Inclusive School}

\author{
$\mathrm{M}^{\mathrm{a}}$ Isabel Calvo * \\ Miguel Ángel Verdugo \\ Antonio Manuel Amor
}

Instituto Universitario de Integración en la Comunidad, Universidad de Salamanca

\begin{abstract}
Las familias han estado habitualmente postergadas de un rol activo en la dinámica educativa. Hoy día, esta situación todavía es una realidad en el momento actual. Para incrementar la participación familiar se han de planificar explícitamente actividades con esa finalidad, especificando con claridad los tipos posibles de participación y definiendo rotundamente el rol que corresponde desempeñar a las familias. Este artículo propone algunas directrices a tener en cuenta para mejorar la participación familiar, la cual se considera un requisito imprescindible para una escuela eficaz.
\end{abstract}

Descriptores: Participación, Familia, Inclusión, Legislación.

Families have been routinely neglected of an active role in the educational dynamics. Nowadays, this situation is still a reality in the present. To increase family involvement activities should be explicitly planned for that purpose, specifying clearly the possible types of participation and defining the role to be played by families. This article proposes some guidelines to consider for enhancing family involvement, which is considered an essential requisite for an inclusive school.

Keywords: Participation, Family, Inclusion, Legislation. 


\section{Introducción}

Hablar de escuela inclusiva inevitablemente lleva consigo hablar de una relación activa y positiva entre todos los agentes involucrados en el proceso educativo, lo que implica diseñar juntos qué es lo mejor para el hijo y/o alumno y entre todos determinar qué educación se quiere y hacia dónde caminar. Ese camino conduce hacia una educación inclusiva en la que todos participan activamente, transformando la escuela en base a los principios de normalización e igualdad de oportunidades, y donde el respeto a la diversidad está presente como valor y principio de la acción. Familia y escuela tienen roles diferentes pero complementarios dirigidos a un objetivo común, pues son dos caras de la misma moneda (Hernández y López, 2006). Sabemos que no es una tarea sencilla, pero es mucho más fácil alcanzar buenos resultados, lograr una plena inclusión y mejorar la calidad de vida del alumnado remando todos en la misma dirección: familia, escuela y comunidad. Hablar de esta relación no debería seguir siendo un reto en este siglo XXI, pues significa hablar de calidad educativa y de educación inclusiva.

El Marco de Acción de la Declaración de Salamanca (1994) dice que "se deberán estrechar las relaciones de cooperación y de apoyo entre los administradores de las escuelas, los profesores y los padres" y "se procurará que estos últimos participen en la adopción de decisiones, en actividades educativas en el hogar y en la escuela y en la supervisión y apoyo del aprendizaje de sus hijos". Además, en el año 2000 en Dakar (Senegal), representantes de organizaciones internacionales y gobiernos del mundo se comprometieron a lograr una "Educación para Todos y Todas" (ETP) en 2015. Ya ha finalizado 2015 y las preguntas a responder, son: ¿Se ha alcanzado este objetivo? ¿Las leyes de educación cumplen el derecho a la Educación para Todos? ¿Qué papel ha jugado la familia en este proceso? ¿Se han creado mecanismos de participación?

La escuela no es el único contexto educativo, sino que la familia, los medios de comunicación y la sociedad en general desempeñan un importante papel en el proceso educativo (Bolívar, 2006). La familia como cualquier otro agente tiene un papel clave en el desarrollo de los niños y jóvenes; nadie puede ni debe encargarse de su educación en exclusiva ni en solitario, todos deben analizar y determinar cuál es su función en el proceso educativo, con el fin de contribuir al desarrollo integral del alumnado dando una respuesta educativa de calidad. La sociedad y la escuela han evolucionado, pero no sólo ellas, sino que la familia también ha sufrido cambios sustanciales: desde una configuración patriarcal o matriarcal a una familia nuclear; de una escasa participación hasta su papel activo y protagonista en la escuela y en las dinámicas internas y educativas relacionadas con sus hijos.

Uno de los objetivos más destacados de las políticas educativas de algunos países occidentales en los últimos años ha sido la de implicar a las familias en la educación y contexto escolar. Del mismo modo, las investigaciones recogen la necesidad de involucrar a las familias como a otros agentes comunitarios, por un lado, para garantizar el éxito educativo (Verdugo y Rodríguez-Aguilella, 2008, 2009) y, por otro, para mejorar aspectos relevantes del funcionamiento de la persona, como son la autodeterminación (Arellano y Peralta, 2015), la calidad de vida individual (Verdugo y Rodríguez-Aguilella, 2009, 2011) y la calidad de vida familiar (Fernández, Montero, Martínez, Orcasitas y Villaescusa, 2015). Este artículo se centra en analizar y orientar diferentes aspectos de la participación familiar que las escuelas han de tener en cuenta. 


\section{Participación}

Si hablamos de participación de la familia en la escuela, conviene recordar que ésta ha vivido distintas etapas. En una primera etapa, la participación era escasa, y la familia y la escuela se mantenían alejadas (i.e., educación no obligatoria y segregada). Posteriormente, en los años 80 del siglo pasado, se pasó por un periodo en el que la familia se sentía cliente de los servicios educativos, lo que les llevó a exigir servicios para satisfacer sus necesidades desde este punto de vista. La participación ha sido y en algunos casos sigue siendo puntual, interesada y ocasional, pero se ha incrementado en los últimos años con una participación activa en el proceso educativo, a través de padres interesados en la gestión de los centros, implicados en la toma de decisiones y preocupados por la educación de sus hijos. Nos encontramos, desde la década de los 90, en una etapa de cooperación, participación e implicación, en la que los padres ejercen su rol de padres. Para formar ciudadanos del siglo XXI, autónomos y responsables que trabajan para alcanzar una sociedad más justa e inclusiva, el papel de las familias ha de pasar de ejercer un rol de clientes a un rol de personas implicadas y comprometidas en el diseño de una escuela para todos y trabajar en la escuela que sueñan y quieren para sus hijos en la que todos -profesionales, familias y comunidad, junto al alumnado- colaboren en su organización, gestión y desarrollo. Que los padres se impliquen depende también de los centros educativos, por lo que desde las escuelas se tiene que hacer partícipes a los padres para que se sientan parte del proceso educativo, fomentado su participación activa.

\section{2. ¿Dónde y cómo participan los padres?}

La presencia de los padres en las decisiones educativas que tienen que ver con sus hijos habitualmente está garantizada, pero se aprecia una falta real de participación (Comellas, 2009) al no estar claramente definido el papel que deben desempeñar. Es frecuente que los padres sean solamente receptores de información y participen ocasional y puntalmente.

Existen variables que ayudan o dificultan la participación como son, entre otras: las políticas, las creencias de las familias, las creencias del profesorado, la percepción del profesorado (sobre las familias, la educación y la escuela), los programas, la cultura, el liderazgo, el interés, factores sociales, barreras de comunicación o experiencias negativas. Todas ellas se deben tener presentes en las propuestas de participación, colaboración e implicación de los padres en la vida del centro. La participación puede ser individual, principalmente asistiendo a reuniones y tutorías; y colectiva, a través de Asociaciones de Madres y Padres (AMPAS), en Escuelas de Padres, en la formación de los profesores o en asociaciones de vecinos. Las AMPAS dan respuesta a algunas de las necesidades de las familias (i.e., información y formación), son un nexo entre las familias y el centro educativo y participan también en actividades extracurriculares, si bien esta participación de la familia tiene en ocasiones poca repercusión.

En la Constitución Española de 1978, se apuesta por la participación de los padres en los órganos de participación de los centros como un derecho y un deber de los mismos ante la educación de sus hijos, tal y como se recoge en el artículo 27.7, dedicado a los derechos y libertades: "los profesores, los padres y, en su caso, los alumnos intervendrán en el control y gestión de todos los centros sostenidos por la Administración con fondos 
públicos, en los términos que la ley establezca”. Por otra parte, el artículo 27.2 recoge que "la educación tendrá por objeto el pleno desarrollo de la personalidad humana en el respeto a los principios democráticos de convivencia y los derechos y libertades fundamentales". Por último, el artículo 27.5 determina que "los poderes públicos garantizan el derecho a todos a la educación mediante una programación general de la enseñanza con participación efectiva de todos los sectores afectados".

Para alcanzar una educación de calidad y formar a ciudadanos comprometidos del siglo XXI, es necesaria la participación de profesorado, alumnado, familias y comunidad. La legislación a nivel internacional y nacional sostiene que atender las necesidades educativas especiales de aquellos alumnos que las presentan, desde el prisma de la educación inclusiva, es obligación de toda la comunidad educativa, siendo la participación activa de los padres esencial a lo largo de todo el proceso de detección, identificación, evaluación y respuesta educativa a este alumnado.

En las leyes educativas se observa un incremento de la presencia de las familias, lo que comporta una mayor participación, reconociéndose este derecho, aunque se ha observado que la participación sigue siendo todavía baja. Le corresponde a las familias participar en la elaboración y revisión del Proyecto Educativo, documento clave en los centros educativos fundamentado en el principio de no discriminación e inclusión, que recoge: las características del entorno cultural y social del centro, los valores, los objetivos y las prioridades de actuación y la respuesta educativa de calidad para todos.

En el sistema educativo español, una forma de participación de las familias es a través de los órganos colegiados. Entre ellos destaca el Consejo Escolar, máximo órgano colegiado que promueve la participación de los padres en la gestión del centro como un derecho y un deber de los mismos: los padres opinan y toman decisiones, reconociéndose por tanto el derecho de las familias a intervenir en los centros educativos. La Ley Orgánica 2/2006, de 3 de mayo, de Educación (LOE), en el artículo 118.5 recoge que "los padres y los alumnos podrán participar también en el funcionamiento de los centros a través de sus asociaciones. Las administraciones educativas favorecerán la información y la formación dirigida a ellos". En relación a las familias con hijos con necesidades educativas, el artículo 71.4, especifica que "corresponde a las Administraciones educativas garantizar la escolarización regular y asegurar la participación de los padres o tutores en las decisiones que afecten a la escolarización y a los procesos educativos de este alumnado" y "adoptar las medidas oportunas para que los padres de estos alumnos reciban el adecuado asesoramiento individualizado, así como la información necesaria que les ayude en la educación de sus hijos".

La actual ley educativa en España, Ley Orgánica 8/2013, de 9 de diciembre, para la Mejora de la Calidad Educativa (LOMCE), convierte a los Consejos Escolares en órganos consultivos, lo que conlleva un cambio en el papel de las familias y disminuye su implicación en la toma de decisiones (art. 1). No debemos olvidar que las últimas leyes reconocen a los padres como los primeros responsables de la educación de sus hijos y, teniendo en cuenta el valor de las familias en el contexto escolar, ¿Avanzamos o retrocedemos? ¿Se han alcanzado los objetivos propuestos en la normativa?

Son distintos los estudios e investigaciones que recogen cómo la participación de las familias en las AMPAS y en los Consejos Escolares es menor de lo que se pretendía, siendo habitual que sean los mismos padres quienes representan a todos y los que acuden a las actividades propuestas. Los datos apuntan que no se logra involucrar a las familias 
en las estructuras de participación, siendo la participación más formal que real (Consejo Escolar del Estado, 2014; Fernández-Enguita, 1993; Informe España de la Fundación Encuentro, 1997; INCE, 1998; Martín-Moreno, 2000; Torres, 2013). Llevot y Bernad (2015) plantean la necesidad de que las AMPA busquen otras estrategias y medios para responder a las necesidades e intereses de las familias.

Por otro lado, existen trabajos que establecen que cuando concurre una relación padresescuela y existe una participación de la familia en la vida escolar, ambos hechos son fundamentales en: la mejora del rendimiento académico del alumnado; una mayor autoestima en los niños; se desarrollan actitudes y comportamientos positivos y se mejora también la calidad de las relaciones interpersonales padres-hijos; se generan actitudes positivas hacia la escuela; y el centro mejora su calidad educativa (Flecha, 2009; Hernández y López, 2006; Includ-ed, 2011; Kñallinsky, 1999; Martínez, 1996; Martínez y Álvarez, 2005; OCDE., 1997; Ordoñez, 2005; Tellado y Sava, 2010; Verdugo y Rodríguez-Aguilella, 2009). Redding (2000) subraya que cuando las familias se relacionan entre sí, el capital social se incrementa y los niños reciben la atención de un número mayor de adultos y los padres comparten pautas, normas y experiencias educativas. Esto implica que hay que construir un espacio de encuentro, una escuela para todos y de todos, donde se refuercen valores comunes a través de la confianza en la familia y en la educación, donde predomine la responsabilidad compartida, colaboración y cooperación respetando el derecho de todos.

El trabajo llevado a cabo por las asociaciones de madres y padres ha dado lugar a distintos modos de participar. Recogemos en la tabla 1 distintas formas de participación partiendo de la propuesta de Bolívar (2006).

Tabla 1. Participación y resultados de la participación

\begin{tabular}{|c|c|c|}
\hline $\begin{array}{c}\text { FORMA DE } \\
\text { PARTICIPACIÓN }\end{array}$ & ACCIONES & RESULTADOS \\
\hline $\begin{array}{l}\text { Mejorar la } \\
\text { articulación de la } \\
\text { educación entre } \\
\text { escuela y familia }\end{array}$ & $\begin{array}{l}\text { Mantener comunicación } \\
\text { fluida familia-escuela: buzón } \\
\text { de sugerencias, agenda } \\
\text { escolar, uso del mail y } \\
\text { plataformas, talleres, obras } \\
\text { de teatro, actividades } \\
\text { extraescolares, jornadas de } \\
\text { puertas abiertas, encuentros } \\
\text { con la naturaleza }\end{array}$ & $\begin{array}{l}\text { Conocer el progreso de sus hijos } \\
\text { Trabajo conjunto } \\
\text { Compromiso } \\
\text { Se fortalecen las relaciones }\end{array}$ \\
\hline $\begin{array}{l}\text { Participación en } \\
\text { la configuración } \\
\text { del centro } \\
\text { educativo }\end{array}$ & $\begin{array}{l}\text { Participar en la elaboración } \\
\text { de los documentos del } \\
\text { centro, conocer, diseñar } \\
\text { normas del centro... }\end{array}$ & $\begin{array}{l}\text { Las familias son un miembro más de la vida } \\
\text { del centro. } \\
\text { Satisfacción e ilusión } \\
\text { Mejores resultados }\end{array}$ \\
\hline $\begin{array}{l}\text { Prestación de } \\
\text { servicios } \\
\text { complementarios } \\
\text { a la escuela }\end{array}$ & $\begin{array}{l}\text { Servicios: escuela de padres } \\
\text { Actividades } \\
\text { extracurriculares }\end{array}$ & $\begin{array}{l}\text { Cubren las necesidades de las familias } \\
\text { Recursos para el centro } \\
\text { Enriquecimiento personal }\end{array}$ \\
\hline $\begin{array}{l}\text { Programas } \\
\text { educativos } \\
\text { comunitarios }\end{array}$ & $\begin{array}{l}\text { Trabajar junto a otras } \\
\text { asociaciones de la comunidad } \\
\text { Nuevos servicios }\end{array}$ & $\begin{array}{l}\text { Educación más práctica y orientada a la vida } \\
\text { Se potencia el conocimiento y cuidado del } \\
\text { entorno } \\
\text { Mejores relaciones comunidad-centro escolar } \\
\text { Inclusión social }\end{array}$ \\
\hline
\end{tabular}

Fuente: Elaboración propia a partir de Bolívar (2006). 
Avanzar hacia una escuela inclusiva, necesariamente nos lleva a aumentar la participación y colaboración de todos los miembros de la comunidad educativa y de las distintas administraciones, aportando cada cual lo mejor de sí: colaboración, compromiso, respeto, recursos, apoyos, información y formación. Hoy por hoy, son las administraciones educativas a las que les corresponde adoptar las medidas oportunas para que los padres de los alumnos que requieran una atención educativa diferente a la ordinaria reciban el adecuado asesoramiento, así como la información necesaria que les ayude en la educación de sus hijos. Los datos aportados por distintos estudios, reclaman a las administraciones públicas una mayor iniciativa y apoyo en los procesos de inclusión (Echeita et al., 2009; Verdugo y Rodríguez-Aguilella, 2008, 2009), pues lo habitual es encontrar barreras que impiden avanzar en los procesos inclusivos en conjunto con las familias y los profesionales comprometidos.

Para que este asesoramiento e información puedan ser eficaces, desde la normativa vigente y las prácticas profesionales es necesaria una mayor actualización. A modo de ejemplo, podemos destacar la normativa de atención a la diversidad en España, pues en diferentes comunidades autónomas puede verse que el enfoque que se da al alumnado con necesidades educativas especiales (e.g., por presentar discapacidad intelectual) está desfasado y el lenguaje y modelos de referencia son inapropiados teniendo en cuenta la Convención de la ONU de 2006 sobre los Derechos de las Personas con Discapacidad (ONU., 2006) o la visión actual de discapacidad (AAIDD., 2010). Así, revisar la terminología incluida en la normativa, proponer indicadores concretos a tener en cuenta con esta población en los procesos de detección, identificación, evaluación y atención educativa, formar a los diferentes agentes de la comunidad educativa, determinar sus responsabilidades en base a indicadores, etc., se antoja esencial para que haya una buena respuesta a estas necesidades y para que la familia pueda desempeñar un papel activo y coherente con el resto de miembros de la comunidad educativa en la educación de sus hijos.

Así, sería adecuado que desde la normativa legal se recogiesen indicadores a considerar a la hora de detectar e intervenir sobre las necesidades que tenga el alumnado, velando por la participación de los padres. Estos indicadores deben guiar la participación de los padres y de otros profesionales no especialistas en atención a la diversidad, haciendo más efectiva la participación de toda la comunidad educativa. Para garantizarlo, se debe especificar los siguientes aspectos:

- Agentes implicados (i.e., padres, tutores, equipo docente, profesionales especialistas, etc.).

- Momentos (atención temprana, detección, identificación, derivación, etc.).

- instrumentos (i.e., observación, entrevistas, cuestionarios, etc.).

- Áreas del desarrollo a considerar (i.e., desarrollo cognitivo, afectivo, social, motor, etc.).

- Contextos (i.e., social, comunitario, educativo, familiar, etc.).

- Medidas de respuesta educativa. Se deben proponer igualmente: agentes (destacando el papel de la familia), momentos, sobre qué áreas y en qué contextos, tipo de medidas más allá de lo curricular (i.e., medidas que aplican 
los padres en casa) y articulación de la coordinación (i.e., para garantizar la participación de todos sin duplicidades).

\section{Comunidad y escuela inclusiva}

Una escuela abierta a la comunidad conlleva no ceder sólo sus instalaciones al barrio, sino que supone implicar a todas las instituciones, organizaciones y grupos de la comunidad en el diseño de proyectos educativos que se pueden generar en el centro y fuera del centro, donde todos participen y se favorezca el desarrollo en los distintos contextos. Caminamos hacia una educación inclusiva donde la acción educativa debe ser tarea compartida por familias, escuela y otras instituciones sociales comprometidas con la educación. ¿Qué papel desempeña la comunidad en este proceso? Resumimos en la tabla 2 los diferentes tipos de participación de la comunidad y su influencia en el aprendizaje y el rendimiento escolar. Se ha subrayado en gris los dos tipos de participación que no desarrollan la inclusión, ni aumentan el rendimiento de los estudiantes (Flecha, García, Gómez y Latorre, 2009).

Tabla 2. Tipos de participación de la comunidad

\begin{tabular}{cl}
\hline \multirow{2}{*}{ INFORMATIVA } & Las familias reciben información sobre las actividades escolares, el \\
& funcionamiento del centro y las decisiones que ya se han tomado. \\
& Las reuniones de padres y madres consisten en informar a las familias sobre \\
& dichas decisiones. \\
& Los padres y madres tienen un poder de decisión muy limitado. \\
CoNSULTIVA & La participación se basa en consultar a las familias. \\
& La participación se canaliza a través de los órganos de gobierno del centro. \\
& Los miembros de la comunidad participan en los procesos de toma de \\
decisiones, teniendo una participación representativa en los órganos de toma de \\
decisión. \\
Lecisoria familias y otros miembros de la comunidad supervisan el rendimiento de \\
cuentas del centro en relación a resultados educativos que obtienen. \\
Las familias y otros miembros de la comunidad participan en el proceso de \\
aprendizaje y del alumnado, ayudando a evaluar sus progresos educativos. \\
Las familias y otros miembros de la comunidad participan en la evaluación \\
general del centro. \\
Las familias y otros miembros de la comunidad participan en las actividades de \\
aprendizaje del alumnado, tanto en horario escolar como extraescolar. \\
Las familias y otros miembros de la comunidad participan en programas \\
educativos que dan respuesta a sus necesidades.
\end{tabular}

Fuente: Recuperada de Arostegui, Darretxe y Beloki (2013).

Como hemos visto, la participación de los padres en el contexto escolar se lleva a cabo a través del Consejo Escolar, a través de comisiones creadas en los centros para su mejor funcionamiento, desde comisiones de convivencia a comisiones económicas y en las AMPAS, involucrándose a distinto nivel. La participación también se produce tanto de manera formal (e.g., entrevistas, reuniones, talleres, proyectos), como informal (i.e., entrada y salida del colegio) y también puntual (i.e., voluntarios en el centro). Asimismo, existen otras alternativas para información e implicar a los padres: murales, pancartas, webs, campañas de sensibilización, anuncios, cuentos o exposiciones. 


\section{Algunas ideas para mejorar la participación familiar en la escuela inclusiva}

El Informe Europeo sobre la Calidad de la Educación Escolar recoge que la participación de los padres en la educación de sus hijos tiene consecuencias políticas en todos los países europeos. Los padres se están convirtiendo en unos consumidores cada vez más exigentes y críticos y pueden contribuir eficazmente a mejorar las escuelas mediante el apoyo a la dirección y profesorado, o pueden impedir que el centro avance y generar conflictos con consecuencias negativas (Comisión Europea., 2000).

Una forma de entender la participación de la familia, es como un recurso para la mejora de los procesos y resultados educativos. Otra, es a través del diseño de programas que implican a la familia y disminuyen las dificultades que pueden darse en esa relación; dificultades derivadas de la cultura, las creencias, la falta de confianza en los profesores, la rivalidad, la diversidad y singularidad de las familias, la diversidad de propuestas y actividades o el tipo de escuela.

Son muchos los trabajos que se centran en cuestiones como: ¿Ha cambiado la relación escuela-familia? ¿Está en crisis la relación familia-escuela? ¿Tienen opinión las familias? ¿Cómo ha sido la comunicación con las familias? (Fernández-Enguita, 2009, Araostegui et al., 2013, Rivas y Ugarte, 2014). Podemos señalar que en la actualidad, la colaboración y participación de las familias y la comunidad se genera a través de proyectos, experiencias y programas de colaboración familia-escuela fuera de lo marcado por la legislación, el Consejo Escolar y las AMPAS. Los beneficios que se derivan de la participación de los padres en la institución han sido muy bien identificados por GarcíaBacete (2003), como puede apreciarse en la tabla 3.

Tabla 3. Efectos de la participación de los padres en la escuela

\begin{tabular}{ll}
\hline EFECTOS EN LOS ESTUDIANTES & \\
Mejores notas, mejores puntaciones en tests de & Realización de los deberes, tenacidad y \\
rendimiento, mayor acceso a estudios de & perseverancia académica \\
postsecundaria & Participación en las actividades de aula \\
Actitudes más favorables hacia las tareas & Menor escolarización en programas de \\
escolares & educación especial \\
Conducta más adaptativa, autoestima más & Menor tasa de abandonos y absentismo, de \\
elevada & suspensión de derechos escolares \\
\hline EFECTOS EN LOS PROFESORES & \\
Los padres les reconocen mejores habilidades & Mayor satisfacción con su profesión \\
interpersonales y de enseñanza & Mayor compromiso con la instrucción (más \\
Los directores valoran más su desempeño & tiempo, más experiencial y centradas en el \\
docente & niño) \\
\hline EFECTOS EN LOS PADRES & Mayor motivación para continuar su propia \\
Incrementan su sentido de autoeficacia & educación \\
Incrementan la comprensión de los programas & Desarrollan habilidades positivas de \\
escolares & paternidad \\
Mejora la comunicación con sus hijos en & Valoran más su papel en la educación de sus \\
general y sobre las tareas escolares en & hijos \\
particular & a \\
\hline Fuente: Elaboración propia a partir de García-Bacete (2003)
\end{tabular}

Fuente: Elaboración propia a partir de García-Bacete (2003).

El estudio de Llevot y Bernad (2015) refleja en relación a la comunicación que se genera entre el centro y la familia, la satisfacción de los implicados en el proceso, señalando también que las familias no conocen lo suficiente el funcionamiento del centro. Ello nos 
lleva a plantear nuevas formas de participación e implicación que desarrolle el interés de los padres y genere un clima de trabajo que favorezca la calidad educativa.

No debemos olvidar que cada contexto escolar y social tiene sus propias características, por lo que es necesario diseñar proyectos y/o actividades innovadoras que permitan a las familias, a la escuela y a la comunidad trabajar juntos generando relaciones de calidad, donde impere la confianza y la comunicación, el respeto, el tiempo compartido, los apoyos y formación recibidos y los recursos de la comunidad. Por tanto, es importante conocer las necesidades del centro, siendo una herramienta de gran utilidad y disponibilidad inmediata el Index for Inclusion (Booth, y Ainscow, 2011), ya en su tercera edición, y traducido en 2015 al Español. El Index permite a los miembros de la comunidad educativa realizar una autoevaluación e identificar las fortalezas y debilidades que tienen respecto a los procesos a implementar, e iniciar así el proceso de inclusión, lo que favorece la reflexión y la acción. En la figura 1 se presentan algunos indicadores del Index que hacen referencia a la relación familia-escuela-comunidad.
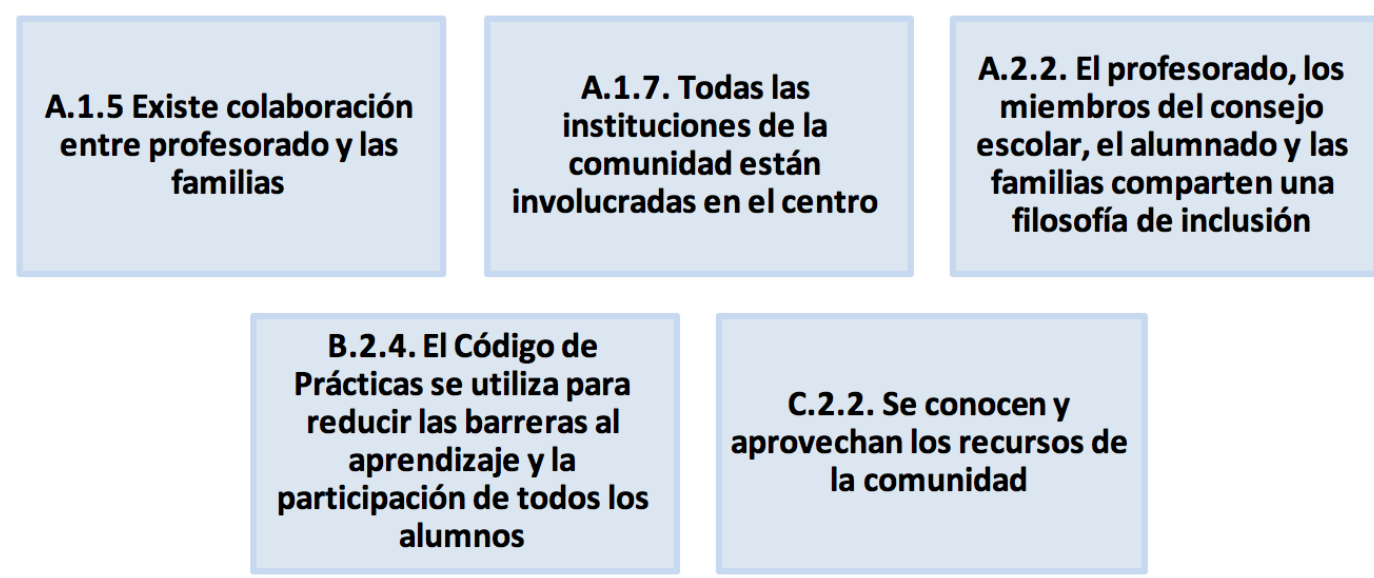

Figura 1. Indicadores de la Guía para la Educación Inclusiva referidos a la familia y el entorno

Fuente: Elaboración propia.

Una propuesta para implicar a la familia y a la sociedad en el contexto escolar son las Comunidades de Aprendizaje. Las Comunidades de Aprendizaje ofrecen una educación de calidad para todas las personas en la Sociedad de la Información y el Conocimiento (García, Lastikka y Petreñas, 2013) y se basan en el aprendizaje dialógico, el cual se entiende como una transformación de la escuela y el contexto social. Los centros se transforman para ofrecer a su alumnado una educación de calidad. La escuela, poco a poco, tiene que permitir la participación activa de las familias en todos los aspectos que concierne a la educación como son los objetivos, valores, métodos de evaluación, recursos y otros aspectos del proceso educativo (Meirieu, 2004). Así, "la necesidad de una relación entre escuela y familia está llevando al desarrollo de nuevas concepciones del aprendizaje que ponen el énfasis en la interacción como el aprendizaje dialógico" (Flecha, 2009, p. 158). En este artículo subrayamos la importancia del dialogo, basándonos en distintas investigaciones que confirman que a través del diálogo se mejora la participación y se reconoce el derecho a opinar de todos los agentes que participan en el proceso educativo. En las Comunidades de Aprendizaje se crean comisiones mixtas de trabajo en las que todos juntos (i.e., personas que se relacionan con 
el alumno) y a través de interacciones dialógicas, deciden lo que se pone en marcha para mejorar la práctica educativa.

En las Comunidades de Aprendizaje todos participan para que se cumpla el sueño, lo que hace que cada vez más las familias y la comunidad dialoguen más y mejor con la escuela. La comunicación escuela-familia se ha basado en actos comunicativos de poder (e.g., el proyecto de centro estaba ya elaborado cuando se presentaba a las familias). Sin embargo, ahora, todos participan en la elaboración e implementación del proyecto. Las familias y miembros de la comunidad participan en grupos interactivos, comisiones mixtas y tertulias dialógicas. En el proceso se ha observado que es necesaria y muy importante la formación en las Comunidades de Aprendizaje, pues surge de las necesidades de las familias y miembros de la comunidad que participan en el proyecto, dando como resultado mayor implicación y compromiso de todos en los centros.

Tanto a nivel nacional como internacional, la participación de los padres es considerada como un aspecto clave en la calidad educativa. El siguiente paso es definir y poner en marcha los mecanismos que favorezcan la participación no sólo de los padres sino de todos los agentes implicados en el proceso. Aquí recogemos algunos indicadores para alcanzar el objetivo:

- Profesores y familias creen que el cambio es posible.

- Diálogo y comunicación entre familia, escuela y comunidad.

- Colaboración, compromiso y responsabilidad en el proceso.

- Formación. Habilidades y conocimientos específicos.

- Todos aportan en el proceso ("todos somos expertos") y todos tienen experiencias que pueden compartir.

- Empatía (ponerse en el lugar del otro).

- Conocer el papel de cada uno: reconocemos que cada uno es diferente y cambia con el paso del tiempo.

- Conexión y coordinación del centro y las familias con otras entidades comunitarias.

- Cada uno es un recurso: las escuelas se convierten en centros de recursos para la comunidad y los miembros de la comunidad son agentes de cambio.

- Detección e implicación activa de las familias en el proceso.

- Crear redes de colaboración dentro y fuera de la escuela como apoyo a la familia: familia extensa, vecinos, servicios sociales, culturales, educativos, redes sociales, tutorías virtuales, con horarios flexibles que se ajusten a las características de las familias.

Cualquier propuesta tendrá en cuenta la diversidad familiar, la opinión, disponibilidad e interés, planificando siempre que sea posible las mismas con la familia (oportunidad de proponer, tomar decisiones, valorar las propuestas del centro,...). Además, serán acciones a corto, medio y largo plazo que permitirán distintos niveles de implicación: 
- Entrevistas, reuniones informativas, reuniones formativas con los padres en grupo-clase en grupos pequeños, según: intereses, necesidades para conocerse, compartir experiencias, conocimientos, evaluación...

- Actividades Complementarias, para implicar a la familia en la organización y planificación, para promover experiencias de intercambio en contextos no formales como recurso de apoyo para aquellas familias que lo necesiten.

- Páginas web, como herramientas de información, noticias y agenda para que se produzca un intercambio de información rápida y eficaz; y blogs como espacios de comunicación abierta y fluida, siempre que las circunstancias familiares, sociales y económicas lo permitan.

- Proyectos de formación. Los padres aprenden para favorecer el desarrollo de su hijo. La familia es un lugar privilegiado para la educación junto con la comunidad y el barrio, por lo que los padres tienen que participar en ellos.

- Programas, proyectos sobre actitudes del profesor, familia y equipo directivo, a través de los que se adquieren valores y actitudes para la convivencia, la colaboración, el trabajo en equipo y el desarrollo individual y colectivo.

- Proyectos de formación del profesorado para que adquieran las competencias que le preparen para trabajar con la familia y los agentes de la comunidad.

\section{Consideraciones finales}

Como se ha visto anteriormente, la participación de las familias en la educación de sus hijos ha pasado por distintos periodos, desde la no participación o participación puntual hasta su implicación activa. Esto ha sido posible gracias a los avances que se han producido en los centros educativos tanto a nivel de gestión y organización, como al cambio de actitud de los implicados. Actualmente, los centros educativos buscan desarrollar nuevas formas de hacer y de ser para responder a los retos de la educación inclusiva. Contamos con modelos, herramientas y estrategias que favorecen una Educación en mayúsculas, una educación para todos, con experiencias positivas y con resultados beneficiosos y eficaces (e.g., Comunidades de Aprendizaje; Guía para la educación Inclusiva / Index for Inclusion).

Los cambios se producen cuando la participación y el compromiso son de todos y todos se sienten parte importante en el proceso, en el cual cada uno aporta según sus habilidades, competencias y funciones. En este proceso de transformación no se debe olvidar que la familia es un recurso vivo que necesita sentirse acogida, tenida en cuenta y que forma parte de la educación de sus hijos. La familia es imprescindible en todos los momentos del proceso. Un aspecto que favorece la convivencia entre escuela y familia es un centro abierto a la comunidad, donde predomine la colaboración. Un funcionamiento eficaz, por tanto, requiere la participación de todos los miembros de la comunidad escolar.

Todavía queda trabajo por hacer en una educación para todos pero ésta ya no será una entelequia, sino una realidad donde la familia, entre otros agentes, juega un papel clave: la participación de la familia es un requisito imprescindible para una escuela inclusiva formando parte de la innovación y la calidad educativa. El objetivo prioritario es 
elaborar políticas inclusivas, crear culturas inclusivas y desarrollar prácticas inclusivas, ampliando las líneas de investigación, evaluando la participación y las relaciones familiaescuela para determinar su repercusión en el aprendizaje, en la implicación y participación en la comunidad.

\section{Referencias}

Arellano, A. y Peralta, F. (2015). Autodeterminación personal y discapacidad intelectual: un análisis desde la perspectiva de las familias. Siglo Cero, 46(3), 255-275.

Arostegui, I., Darretxe, L. y Beloki, N. (2013). La participación de las familias y de otros miembros de la comunidad como estrategia de éxito en las escuelas. Revista Iberoamericana de Evaluación Educativa, 6(2), 187-200.

Bolívar, A. (2006). Familia y escuela: dos mundos llamados a trabajar en común. Revista de Educación, 39, 119-146.

Booth, T. y Ainscow. M. (2011). Index for inclusion. Developing learning and participation in schools. Bristol: CSIE.

Comisión Europea. (2000). Informe europeo sobre la calidad de la educación escolar. Dieciséis indicadores de calidad. Luxemburgo: Oficina de Publicaciones Oficiales de las Comunidades Europeas.

Comellas, M. J. (2009). Familia y escuela: compartir la educación. Barcelona: Grao.

Consejo Escolar del Estado. (2014). La participación de las familias en la educación escolar. Madrid: Ministerio de Educación, Cultura y Deporte.

Echeita, G., Simón, C., Verdugo, M. A., Sandoval, M., López, M., Calvo, M. I. y González, F. (2009). Paradojas y dilemas en el proceso de inclusión educativa en España. Revista de Educación, 349, 153-178.

Feito, R. (1991). La participación de los padres en el control y gestión de la enseñanza (Tesis doctoral). Universidad Complutense de Madrid. Madrid.

Fernández, A., Montero, D., Martínez, N., Orcasitas, J. R. y Villaescusa, M. (2015). Calidad de vida familiar: marco de referencia, evaluación e intervención. Siglo Cero, 46(2), 254, 7-29.

Fernández-Enguita, M. (1993). La profesión docente y la comunidad escolar. Crónica de un desencuentro. Madrid: Morata.

Fernández-Enguita, M. (2005). Organización escolar, profesión docente y entorno comunitario. Madrid: Akal.

Flecha R. (2009). Cambio, inclusión y calidad en las comunidades de aprendizaje. Cultura y Educación, 21(2), 157-169. doi:10.1174/113564009788345835

Flecha, A., García, R., Gómez, A. y Latorre, A. (2009). Participación en escuelas de éxito: una investigación comunicativa del proyecto Includ-ed. Cultura y Educación, 21(2), 183-196. doi: $10.1174 / 113564009788345899$

Fundación Encuentro. (1997). Informe España 1996. Una interpretación de su realidad social. Madrid: Fundación Encuentro.

García, C., Lastikka, A. L. y Petreñas, C. (2013). Comunidades de aprendizaje. Scripta NovaRevista Electrónica de Geografía y Ciencias Sociales, 17(4), 27-43.

García-Bacete, F. J. (2003). Las relaciones escuela-familia: un reto definitivo. Infancia y Aprendizaje, 26(4), 425-437. doi:10.1174/021037003322553824 
Hernández Prados, M. A. y López Lorca, H. (2006). Análisis del enfoque actual de la cooperación padres y escuela. Aula Abierta, 87, 3-26.

INCE. (1998). Familia y escuela. Madrid: Ministerio de Educación y Cultura.

Includ-ed. (2011). Actuaciones de éxito en las escuelas europeas. Madrid: IFIE.

Kñallinsky, E. (1999). La participación educativa: familia y Escuela (Tesis doctoral). Universidad de Las Palmas de Gran Canaria, Las Palmas de Gran Canaria.

Llevot, N. y Bernand, O. (2015). La participación de las familias en la escuela: factores clave. Revista de la Asociación de Sociología de la Educación. Revista de la Asociación de Sociología de la Educación, 8(1), 57-70.

Martín, M. y Gairín, J. (2007). Familias en la educación: un tema por resolver. Bordón, 59(1), 113151

Martín-Moreno, Q. (2000). Bancos de talento. Participación de la comunidad en los centros docentes. Madrid: Sanz y Torres.

Martínez, R. A. y Álvarez, L. (2005). Fracaso y abandono escolar en educación Secundaria Obligatoria: implicación de la familia y los centros escolares. Aula Abierta, 85, 127-146.

Martínez, R. A. (1996). Familia y educación. Oviedo: Servicio de Publicaciones Universidad de Oviedo.

Meirieu, P. (2004). En la escuela hoy. Barcelona: Octaedro.

Navaridas, F. y Raya, E. (2012). Indicadores de participación de los padres en el sistema educativo: un nuevo enfoque para la calidad educativa. Revista Española de Educación Comparada, 20, 223-248. doi:10.5944/reec.20.2012.7599

OCDE. (1997). Parents as partners in schooling. París: Centre for Educational Research and Innovation.

ONU. (2006). Convención de las Naciones Unidas sobre los derechos de las personas con discapacidad. Recuperado de http://www.un.org/spanish/disabilities/

Ordóñez, R. (2005). Medios para mejorar la relación entre la familia y la escuela. Kikiriki. Cooperación Educativa, 78, 38-42.

Pérez-Díaz, V., Chuliá, E. y Valiente, C. (2000). La familia española en el año 2000: innovación y respuesta de las familias a sus condiciones económicas, políticas y culturales. Madrid: Fundación Argentaria.

Pérez-Díaz, V., Rodríguez, J. C. y Fernández, J. J. (2009). Educación y familia. Los padres ante la educación general de sus hijos en España. Madrid: Fundación de las Cajas de Ahorros.

Redding, S. (2000). Familias y escuelas. Ginebra: Academia Internacional de Educación, Bélgica, y la Oficina Internacional de Educación.

Rivas, S. y Ugarte, C. (2014). Formación docente y cultura participativa del cetro educativo: claves para favorecer la participación familia-escuela. ESE. Estudios sobre Educación, 27, 153-168. doi:10.15581/004.27.153-168

Schalock, R. L., Borthwick-Duffy, S. A., Bradley, V. J., Buntinx, W. H. E., Coulter, D., Craig, E. M., ..., Yeager, M. H. (2010). Intellectual disability. Definition, classification, and systems of supports. Washington, DC: American Association on Intellectual and Developmental Disabilities.

UNESCO. (1994). Declaración de Salamanca y marco de acción ante las necesidades especiales. París: UNESCO. 
Tellado, I. y Sava, S. (2010). The role of non-expert adult guidance in the dialogic construction of knowledge. Revista de Psicodidáctica, 15(2), 163-176.

Torres, M. (2013). El capital social en las asociaciones de madres y padres: Formación, desarrollo e institucionalización. Granada: Universidad de Granada.

Verdugo, M. A., Arias, B., Gómez, L. E. y Schalock, R. L. (2010). Development of an objective instrument to assess quality of life in social services: reliability and validity in Spain. International Journal of Clinical Health E Psychology, 1O(1), 105-123.

Verdugo, M. A. y Rodríguez-Aguilella, A. (2008). Valoración de la inclusión educativa desde diferentes perspectivas. Siglo Cero, 39(4), 5-25.

Verdugo, M. A. y Rodríguez-Aguilella, A. (2009). La inclusión educativa en España desde la perspectiva de alumnos con discapacidad intelectual, de familias y de profesionales. Revista de Educación, 358, 450-470.

Verdugo, M. A. y Rodríguez-Aguilella, A. (2011). Guía F. Guía de intervención y apoyo a las familias de personas con discapacidad. Sevilla: Junta de Andalucía.

\section{Breve CV de los autores}

\section{M$^{\mathrm{a}}$ Isabel Calvo Álvarez}

Profesora Contratada Doctor del Departamento de Didáctica, Organización y Métodos de Investigación. Licenciada en Filosofía y Ciencias de la Educación, secc. Ciencias de la Educación y Doctora por la Universidad de Salamanca. Directora del Máster Universitario de Investigación en Discapacidad de la USAL y Miembro del Instituto Universitario de Integración en la Comunidad de la Universidad de Salamanca (INICO). Líneas de investigación relacionadas con Educación, Inclusión, Discapacidad y Calidad de Vida. Publicaciones centradas en las líneas de investigación.

\section{Miguel Ángel Verdugo Alonso}

Catedrático de Psicología de la Discapacidad (Universidad de Salamanca), director del Instituto Universitario de Integración en la Comunidad y del "Máster en Integración de Personas con Discapacidad. Calidad de vida", así como del Servicio de Información sobre Discapacidad del Ministerio de Sanidad, Servicios Sociales e Igualdad y la Universidad de Salamanca. Ha publicado más 500 artículos científicos o capítulos de libros, y más de 70 libros y escalas de evaluación. Director de la Revista Siglo Cero. Ha dirigido investigaciones sobre calidad de vida, discapacidad intelectual, empleo, habilidades sociales y otras. Ha participado frecuentemente como profesor invitado en universidades de diferentes países, y presentado numerosas ponencias y comunicaciones científicas.

\section{Antonio Manuel Amor González}

Licenciado en Psicología por la Universidad de Salamanca. Es Máster en Formación del Profesorado de Educación Secundaria Obligatoria, Bachillerato, Formación Profesional y Enseñanza de Idiomas (sección Orientación Educativa) por la Valencian International University y Máster en Integración de Personas con Discapacidad-Calidad de Vida por la Universidad de Salamanca. Actualmente es contratado predoctoral FPU por el 
Ministerio de Educación, Cultura y Deporte y realiza la tesis doctoral en la Universidad de Salamanca, sobre evaluación y análisis diferencial de necesidades de apoyo en estudiantes con y sin discapacidad intelectual. 